\title{
Counterflow in Bose gas bilayers: Collective modes and dissipationless drag
}

Cite as: Fiz. Nizk. Temp. 46, 572-577 (May 2020); doi: 10.1063/10.0001051

Submitted: 23 March 2020

Saeed H. Abedinpour ${ }^{1,2}$ (D) and B. Tanatar ${ }^{3, a)}$ (iD

\section{AFFILIATIONS}

${ }^{1}$ Department of Physics, Institute for Advanced Studies in Basic Sciences (IASBS), Zanjan 45137-66731, Iran

${ }^{2}$ School of Nano Science, Institute for Research in Fundamental Sciences (IPM), Tehran 19395-5531, Iran

${ }^{3}$ Department of Physics, Bilkent University, Ankara 06800, Turkey

a) Author to whom correspondence should be addressed: tanatar@fen.bilkent.edu.tr

\begin{abstract}
We investigate the collective density oscillations and dissipationless drag effect in bilayer structures of ultra-cold bosons in the presence of counterflow. We consider different types of inter-particle interactions and obtain the drag coefficient and effect of counterflow on the sound velocity. We observe that counterflow enhances (suppresses) the energy of symmetric (asymmetric) density mode and drives the homogeneous system towards instability. The dependence of the drag coefficient on the spacing between two layers is determined by the form of particle-particle interaction.
\end{abstract}

Published under license by AIP Publishing. https://doi.org/10.1063/10.0001051

\section{INTRODUCTION}

Experimental advances in trapping and cooling atoms and ions in low dimensional geometries together with the ability to manipulate different system parameters have provided a unique playground for the simulation of exotic model systems. ${ }^{1-3}$ In particular, the possibility of having different types of particle-particle interactions together with their tunability has made it possible to hunt for interesting physical phenomena, hardly observable in natural conditions. ${ }^{4}$

If trapped particles are bosons, at low enough temperatures one would expect Bose-Einstein condensation $(\mathrm{BEC})^{5,6}$ to take place. Interesting phenomena are expected in multi-component condensates due to the interplay between superfluidity and interparticle interactions.

In this paper, we have considered a bilayer system of Bose gas at zero temperature. Bosons in each layer are in the BEC state. Particles in two separated layers are coupled through the interlayer interaction. The interaction induced depletion of the condensate is not significant, as long as the interlayer and intralayer interactions are not too strong. As a two-component system, two collective density modes are expected for the bilayer structure corresponding to the in-phase and out-of-phase oscillation of density in two layers. If the inter-particle interaction is long-ranged the in-phase collective mode is a plasmon mode with the usual $\omega \propto \sqrt{q}$ long-wavelength dispersion, ${ }^{7}$ while the out-of-phase mode is acoustic with the $\omega \propto q$ dispersion. For short-range interactions both modes become acoustic. Driving one of the two layers with a uniform background velocity, supercurrents flow in both layers. ${ }^{8-18}$ The relative velocity between two layers modifies the dispersions of collective modes, enhancing the energy of in-phase oscillations and softening of the out-of-phase mode. If the counterflow is strong enough, the energy required to excite the out-of-phase mode becomes zero, indicating the instability of homogenous Bose gas towards a density wave or a phase-separated state.

The rest of this paper is organized as follows. In Sec. 2. we introduce our model of double-layer Bose gas and explain how it is possible to obtain its collective density modes and superfluid drag response. In Sec. 3. we present our results for the collective modes and drag effect considering three different forms of particle-particle interactions, namely Coulomb, dipolar, and soft-core interactions. Finally, we summarize and conclude our main findings in Sec. 4.

\section{DENSITY-DENSITY RESPONSE FUNCTION AND COLLECTIVE MODES}

We consider two identical two-dimensional planes of ultracold bosons, separated by a distance $d$ (see, Fig. 1). No tunneling is allowed between two layers. Therefore, layers are coupled together only through the inter-particle interaction. The density fluctuations 


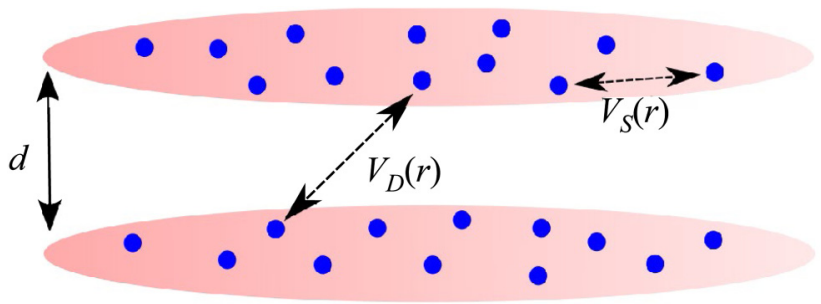

FIG. 1. Cartoon of two identical layers of ultra-cold bosons separated by distance $d$. Particles in each layer interact through $V_{S}(r)$, while $V_{D}(r)$ is interaction between two particle from different layers, $r$ being the in-plane distance between particles.

$\delta n_{i}(\mathbf{q}, \omega)$ in layer $i=1,2$, is given by ${ }^{7}$

$$
\delta n_{i}(\mathbf{q}, \omega)=\sum_{j} \chi_{i j}(\mathbf{q}, \omega) V_{j}^{e x t}(\mathbf{q}, \omega)
$$

where $V_{j}^{\text {ext }}(\mathbf{q}, \omega)$ is the external potential applied to layer $j$ and $\chi_{i j}(\mathbf{q}, \omega)$ is the density-density linear response function, written in the matrix form as

$$
\chi(\mathbf{q}, \omega)=\left[I-W^{\mathrm{eff}}(\mathbf{q}, \omega) \Pi(\mathbf{q}, \omega)\right]^{-1} \Pi(\mathbf{q}, \omega) .
$$

Here, $I$ is a $2 \times 2$ identity matrix, and $W_{i j}^{\text {eff }}(\mathbf{q}, \omega)$ and $\Pi_{i j}(\mathbf{q}, \omega)$ are the elements of the dynamical effective potential and non-interacting density-density response function, respectively. The exact form of the effective potentials are not known, and one has to resort to some approximations. Within the random phase approximation (RPA), one replaces the effective interaction with the bare one $V_{\mathrm{ij}}(q)$. For a symmetric bilayer we have $V_{i j}(q)=\delta_{i j} V_{S}(q)+\left(1-\delta_{i j}\right) V_{D}(q)$, where $V_{s}(q)$ and $V_{\mathrm{D}}(q)$ are the bare interaction between bosons in the same and different layers, respectively. Eigenvalues of the density-density response matrix $\chi(\mathbf{q}, \omega)$ are

$$
\chi_{ \pm}^{R P A}(\mathbf{q}, \omega)=\frac{\Pi(\mathbf{q}, \omega)}{1-V_{ \pm}(q) \Pi(\mathbf{q}, \omega)}
$$

where $V_{ \pm}(q)=V_{\mathrm{S}}(q) \pm V_{\mathrm{D}}(q)$ are the symmetric and antisymmetric components of the interaction, and the non-interacting densitydensity response function of a two-dimensional system of bosons at zero temperature is analytically known

$$
\Pi(q, \omega)=\frac{2 n \varepsilon_{q}}{(\hbar \omega+\mathrm{i} 0+)^{2}-\varepsilon_{q}^{2}},
$$

with $n$ the particle density in each layer and $\varepsilon_{q}=\hbar^{2} q^{2} /(2 m)$ the single-particle energy dispersion of bosons with mass $m$. The dispersion of the collective modes could be obtained from the singularities of the density-density response functions $\chi_{ \pm}(q, \omega)$ at finite frequency. Using the analytic form of the non-interacting density-density response function, it is possible to find analytic expressions for the collective modes' dispersions

$$
\hbar^{2} \omega_{ \pm}^{2}(q)=\varepsilon_{q}^{2}+2 n \varepsilon_{q} V_{ \pm}(q),
$$

which correspond to the in-phase (+) and out-of-phase (-) oscillations of the particle density in two layers.

\subsection{Counterflow}

If we consider background velocities of $\mathbf{v}_{1}$ and $\mathbf{v}_{2}$ in the first and second layers, respectively the collective modes in the presence of these background velocities could be easily obtained from the poles of the total density-density response, after replacing $\omega$ with $\omega-\mathbf{v}_{i} \cdot \mathbf{q}$ in the non-interacting density response of layer $i$ : $\Pi_{i}(q$, $\omega)$. The back-ground flows could be decoupled into the center-of-mass $\mathbf{V}=\left(\mathbf{v}_{1}+\mathbf{v}_{2}\right) / 2$ and counterflow $\mathbf{v}=\left(\mathbf{v}_{1}-\mathbf{v}_{2}\right) / 2$ components. As the effect of the center-of-mass flow could be simply understood in terms of a Galilean boost, we focus on the counterflow part, and look for the solutions of the following equation:

$$
\left|\begin{array}{cc}
\Pi^{-1}(q, \omega-\mathbf{v} \cdot \mathbf{q})-V_{S}(q) & -V_{D}(q) \\
-V_{D}(q) & \Pi^{-1}(q, \omega+\mathbf{v} \cdot \mathbf{q})-V_{S}(q)
\end{array}\right|=0,
$$

which after some straightforward algebra, for the dispersions of collective modes in the presence of finite counter-flow result in

$$
\begin{aligned}
\hbar^{2} \omega_{ \pm}^{2}(\mathbf{q}, \mathbf{v})= & \varepsilon_{q}^{2}\left[1+\frac{2 n}{\varepsilon_{q}} V_{S}(q)\right]+(\hbar \mathbf{v} \cdot \mathbf{q})^{2} \\
& \pm 2 \varepsilon_{q} \sqrt{n^{2} V_{D}^{2}(q)+(\hbar \mathbf{v} \cdot \mathbf{q})^{2}\left[1+\frac{2 n}{\varepsilon_{q}} V_{S}(q)\right]}
\end{aligned}
$$

We note that the dispersions of collective modes become anisotropic in the presence of finite counterflow and for small counterflow velocities, to leading order in $v$ we find

$$
\hbar \omega_{ \pm}(\mathbf{q}, \mathbf{v}) \approx \hbar \omega_{ \pm}(q)+\frac{1}{2} \mathcal{M}_{ \pm}(q, \phi) v^{2}
$$

where

$$
M_{ \pm}(q, \phi)=\frac{\hbar q^{2} \cos ^{2}(\phi)}{\omega_{ \pm}(q)}\left\{1 \pm \frac{\varepsilon_{q}}{n V_{D}(q)}\left[1+\frac{2 n}{\varepsilon_{q}} V_{S}(q)\right]\right\} .
$$

Here, $\omega_{ \pm}(q)$ are the dispersions of collective modes in the absence of counterflow, as given by Eq. (5) and $\phi$ is the angle between the flow direction and the direction of wave vector $\mathbf{q}$.

\subsection{Zero-point energy and drag effect}

With the full dispersion relations of collective excitations, we are able to find the change in the zero-point energy (per unit area), due to the finite counterflow ${ }^{9}$

$$
\Delta E_{Z P}(v)=\frac{\hbar}{2 A} \sum_{q, \alpha= \pm}\left[\omega_{\alpha}(\mathbf{q}, \mathbf{v})-\omega_{\alpha}(q)\right]
$$


in which the difference between collective modes with and without counterflow are summed and $A$ is the sample area. For small counterflow velocities, we can write

$$
\Delta E_{Z P}(v) \approx-2 \gamma_{D} v^{2},
$$

where

$$
\gamma_{D}=-\frac{1}{8 A} \sum_{q, \alpha= \pm} \mathcal{M}_{\alpha}(q, \phi) .
$$

As we will see in the next section, the zero-point energy $\Delta E_{Z P}(v)$ is negative which means that finite counterflow lowers the free energy. We now construct the zero-temperature free energy $F$, by adding the kinetic energies of the bosons in each layer

$$
F=\frac{1}{2} n m\left(v_{1}^{2}+v_{2}^{2}\right)-\frac{\gamma_{D}}{2}\left(v_{1}-v_{2}\right)^{2}
$$

where we have reverted to use the individual velocities in each layer. We find the current densities in layer 1 and layer 2, calculated from $j_{i}=\partial F / \partial v_{i}(i=1,2)$, to be

$$
\begin{aligned}
& j_{1}=\left(n m-\gamma_{D}\right) v_{1}+\gamma_{D} v_{2}, \\
& j_{2}=\gamma_{D} v_{1}+\left(n m-\gamma_{D}\right) v_{2} .
\end{aligned}
$$

The expressions given in Eq. (14) demonstrate that the superflow in the first (second) layer depends on the superfluid velocity on the same layer as well as that of the second (first) layer. This is the dissipationless superfluid drag effect well known in two-component superfluids $^{8-14,17,18}$ which has been discussed for a variety of related systems.

In the following, we will investigate the long-wave-length dispersion of collective modes and the drag coefficient $\gamma_{D}$ for dipolar systems of bosons interacting with different forms of interactions.

\section{RESULTS}

\subsection{Charged bosons}

Bilayers of charged bosons interacting through Coulomb potential has been extensively explored in the literature. Here, we reconsider this system as a matter of completeness and to compare its results with the ones we find for other forms of interaction. For charged bosons, in the real space, we have

$$
\begin{aligned}
& V_{S}(r)=\frac{e^{2}}{r}, \\
& V_{D}(r)=\frac{e^{2}}{\sqrt{r^{2}+d^{2}}},
\end{aligned}
$$

where $r$ is the in-plane distance between two bosons and $d$ is the layer separation. The Fourier transform of the bare interactions read

$$
\begin{aligned}
& V_{S}(q)=\frac{2 \pi e^{2}}{q}, \\
& V_{D}(q)=V_{S}(q) e^{-q d} .
\end{aligned}
$$

The full dispersion of collective modes in the presence of counterflow is obtained from Eq. (7), after replacing the specific form of intralayer and interlayer interactions there-in. In Fig. 2 we have compared the dispersions of in-phase and out-of-phase collective density modes at finite counterflow with the ones in the absence of counterflow. The dimensionless coupling strength is defined as $g_{C}=\pi n a_{B}^{2}$, where $a_{B}=\hbar^{2} /\left(m e^{2}\right)$ is the Bohr radius and the dimensionless velocity is $\tilde{v}=m a_{B} v / \hbar$.

In the long-wavelength limit and in the absence of counterflow the dispersions of collective modes read

$$
\begin{aligned}
& \omega_{+}(q) \approx \sqrt{\frac{4 \pi n e^{2}}{m}} q, \\
& \omega_{-}(q) \approx \sqrt{\frac{2 \pi n e^{2} d}{m}} q=v_{s} q,
\end{aligned}
$$

where $v_{s} \equiv \sqrt{2 \pi n e^{2} d / m}$ is the sound velocity of the charged system. With finite counterflow, the plasmon mode is not affected by the counterflow to the leading order terms in $q$, but the sound velocity is modified as

$$
v_{s}(v, \phi)=\sqrt{v_{s}^{2}-v^{2} \cos ^{2} \phi}
$$

Replacing the long wavelength dispersion of collective modes in

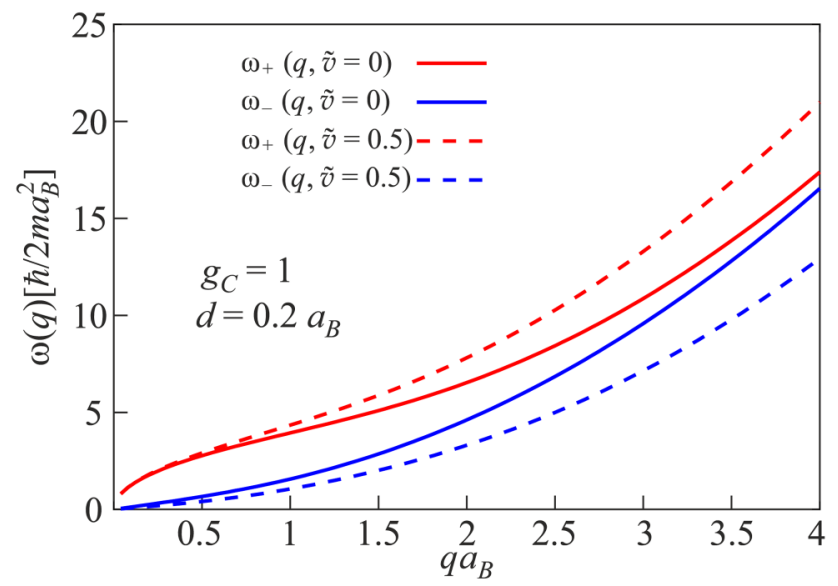

FIG. 2. Dispersions of in-phase and out-of-phase collective density modes in a bilayer of charged bosons in the absence (solid lines) and presence (dashed lines) of finite counterflow velocity. The dispersions are plotted along the flow direction i.e., $\mathbf{q} \| \mathbf{v}$. The dimensionless density parameter is $g_{C}=\pi n a_{B}^{2}$ and the dimensionless velocity is defined as $\tilde{v}=m a_{B} v / \hbar$. 
Eq. (9) the drag coefficient, to leading order in the wave vector, reads

$$
\gamma_{D}=\frac{\hbar}{8 A v_{s}} \sum_{\mathbf{q}} q \cos ^{2} \phi \approx \frac{\hbar}{96 \pi v_{s} d^{3}},
$$

where the expression in the second line is obtained after integrating $q$ up to $1 / \mathrm{d}$. As $v_{s} \propto d^{1 / 2}$, we find $\gamma_{D} \propto d^{-7 / 2}$ at small layer separations. This agrees with the findings of Tanatar and Das in Ref. 10.

\subsection{Dipolar bosons}

If we consider a bilayer loaded with dipolar bosons, whose dipolar moments are aligned perpendicular to the plane, the bare intralayer and interlayer interactions, respectively read ${ }^{19}$

$$
\begin{aligned}
& V_{S}(r)=\frac{C_{d d}}{4 \pi} \frac{1}{r^{3}} \\
& V_{D}(r)=-\frac{C_{d d}}{4 \pi} \frac{r^{2}-2 d^{2}}{\left(r^{2}+d^{2}\right)^{5 / 2}}
\end{aligned}
$$

where $C_{\mathrm{dd}}$ is the dipole-dipole coupling constant, and the direction of polarization in two layers is considered apposite to each other, in order to avoid binding of dipoles from different layers. ${ }^{25}$ In practice, such a configuration could be realized with ultra-cold polar molecules subjected to an external static electric field applied to polarize the dipoles. One would then excite molecules in two adjacent layers into two different rotational states, such that their effective polarization become respectively parallel and antiparallel to the applied external electric field. ${ }^{19,26}$ Upon the Fourier transformation of Eq. (20), we find ${ }^{20,21}$

$$
\begin{aligned}
& V_{S}(q)=\frac{C_{d d}}{4}\left[\frac{8}{3 \sqrt{2 \pi} w}-2 q \mathrm{e}^{q^{2} w^{2} / 2} \operatorname{erfc}\left(\frac{q w}{\sqrt{2}}\right)\right], \\
& V_{D}(q)=\frac{C_{d d}}{2} q \mathrm{e}^{-q d},
\end{aligned}
$$

where $\operatorname{erfc}(x)$ is the complementary error function and $w$ is the short distance cut-off introduced to heal the divergence of Fourier transform of the intralayer interaction. In Fig. 3 we compare the full dispersions of collective density modes of dipolar bosons in bilayer structure at finite and zero counterflows. The dimensionless coupling strength is defined as $g_{D}=\pi n r_{0}^{2}$, where $r_{0}=m C_{d d} /\left(4 \pi \hbar^{2}\right)^{22}$ is the dipole length and the dimensionless velocity is $\tilde{v}=m r_{0} v / \hbar$.

Note that in the long wavelength limit, we have $V_{S}(q \rightarrow 0)$ $\approx U_{0}-C_{d d} q / 2$ and $V_{D}(q \rightarrow 0) \approx C_{d d} q / 2$, where $U_{0}=\sqrt{2 / \pi} C_{d d} /(3 w)$. The dispersions of collective modes at long wavelength read

$$
\omega_{ \pm}(q) \approx v_{s} q+\cdots,
$$

where $v_{s}=\sqrt{n U_{0} / m}$. This shows that both symmetric and asymmetric modes are sound waves with the same zero-sound velocity within the RPA. Finite counterflow breaks the degeneracy between two modes, and the sound velocities are modified as

$$
v_{s, \pm}(v, \phi)=v_{s} \pm v|\cos \phi| .
$$

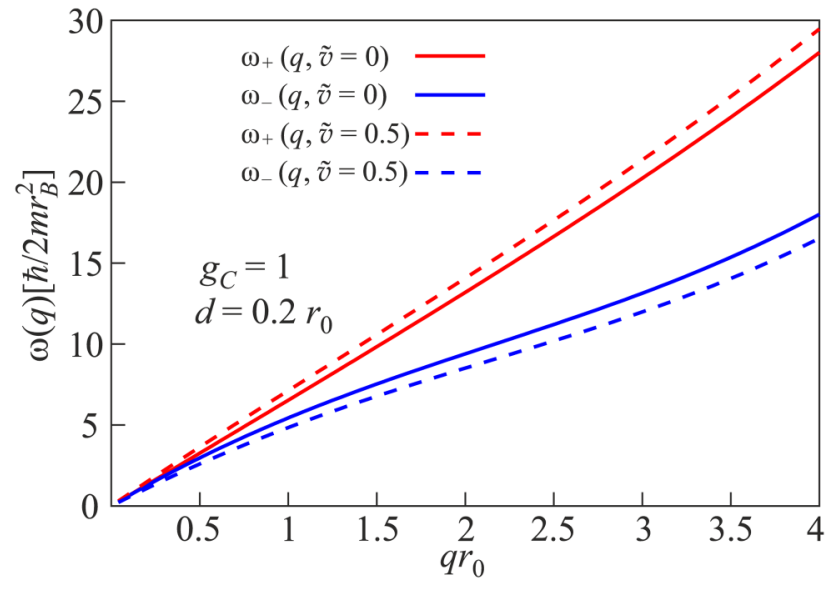

FIG. 3. Dispersions of in-phase and out-of-phase collective density modes in a bilayer of dipolar bosons in the absence (solid lines) and presence (dashed lines) of finite counterflow velocity. The dispersions are plotted along the flow direction i.e., $\mathbf{q} \| \mathbf{v}$. The dimensionless density parameter is $g_{D}=\pi n r_{0}^{2}$ with $r_{0}=m C_{d d} /\left(4 \pi \hbar^{2}\right)$ and the dimensionless velocity is defined as $\tilde{v}=m r_{0} v / \hbar$. Moreover, we have used $w=0.2 r_{0}$ as the cutoff parameter for the intralayer interaction.

The drag coefficient, to leading order in the wave vector, is

$$
\begin{aligned}
\gamma_{D} & \approx \frac{\hbar C_{d d}^{2}}{\mathrm{~A} 64 v_{s} U_{0}^{2}} \sum_{q} q^{3} \cos ^{2} \phi \\
& \approx \frac{\hbar C_{d d}^{2} n^{2}}{1280 \pi m^{2} v_{s}^{5} d^{5}},
\end{aligned}
$$

where, again, $q$ is integrated up to $1 / d$ in the second line.

\subsection{Soft-core interactions}

Now, we consider a bilayer system of particles interacting through a soft-core short-range interaction. This form of interaction is relevant e.g., for Rydberg-dressed particles. ${ }^{23}$ We model this interaction with a step function $U \Theta\left(r_{c}-r\right)$, where $U$ is the strength of interaction and $r_{c}$ is its range. The intralayer and interlayer interactions read

$$
\begin{aligned}
& V_{S}(r)=U \Theta\left(r_{c}-r\right), \\
& V_{D}(r)=U \Theta\left(r_{c}-\sqrt{r^{2}+d^{2}}\right) .
\end{aligned}
$$

Obviously, two layers are decoupled if $d>r_{c}$, therefore we consider only cases where the spacing between two layers is smaller than the range of interaction. In the Fourier space, we find

$$
\begin{aligned}
& V_{S}(q)=2 \pi U r_{c} \frac{J_{1}\left(r_{c} q\right)}{q} \\
& V_{D}(q)=2 \pi U \sqrt{r_{c}^{2}-d^{2}} \frac{J_{1}\left(\sqrt{r_{c}^{2}-d^{2}} q\right)}{q} .
\end{aligned}
$$




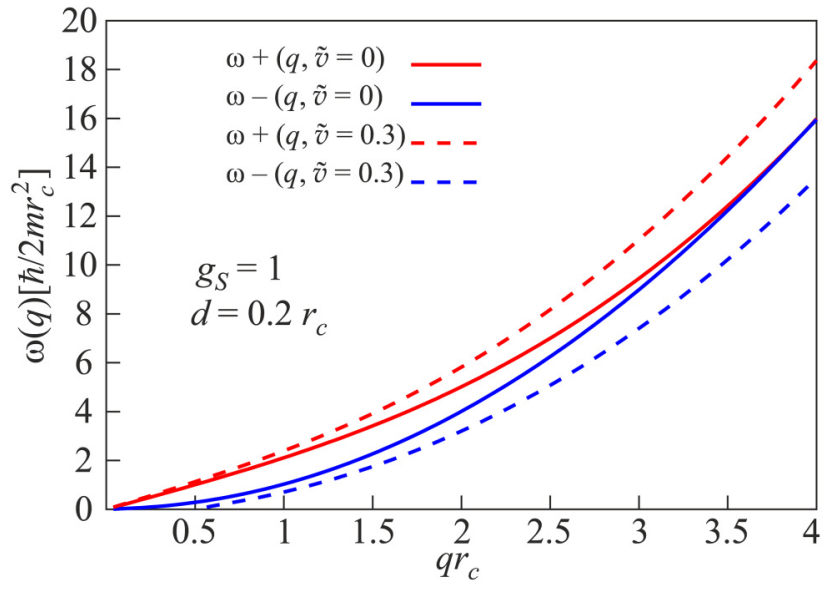

FIG. 4. Dispersions of in-phase and out-of-phase collective density modes in a bilayer of soft-core bosons in the absence (solid lines) and presence (dashed lines) of finite counterflow velocity. The dispersions are plotted along the flow direction, i.e., $\mathbf{q} \| \mathbf{v}$. The dimensionless density parameter is $g_{S}=2 \pi m n r_{c}^{4} U / \hbar^{2}$ and the dimensionless velocity is defined as $\tilde{v}=m r_{c} v / \hbar$.

Here, $J_{1}(x)$ is the Bessel function of the first kind. In the long wavelength limit we have $V_{D}(q=0) \equiv V_{D, 0}=\pi U\left(r_{c}^{2}-d^{2}\right)$ and $V_{S}(q=0) \equiv V_{S, 0}=\pi U r_{c}^{2}$. The collective modes in the absence of counterflow are acoustic with different sound velocities

$$
\omega \pm(q)=v_{s, \pm} q
$$

with $v_{s, \pm}=\sqrt{n\left[V_{S, 0} \pm V_{D, 0}\right] / m}$. It is interesting to note that $v_{s,-}$ $=d \sqrt{n \pi U / m}$ is independent of the soft-core radius $r_{c}$, and linearly increases with the layer spacing $d$ for $d<r_{c}$. With finite counterflow, to leading order contribution from the flow velocity, we find

$$
v_{ \pm}^{2}(v, \phi)=v_{s, \pm}^{2}+v^{2} \cos ^{2} \phi\left[1 \pm 2 \frac{V_{S, 0}}{V_{D, 0}}\right] .
$$

Finally, the drag coefficient reads

$$
\gamma_{D}=\frac{\hbar}{8 A} \sqrt{\frac{m}{n}} G \sum_{q} q \cos ^{2} \phi \approx \frac{\hbar}{96 \pi d^{3}} \sqrt{\frac{m}{n}} G,
$$

where

$$
\begin{aligned}
G & =\frac{2 V_{S, 0}-V_{D, 0}}{V_{D, 0} \sqrt{V_{S, 0}-V_{D, 0}}}-\frac{2 V_{S, 0}+V_{D, 0}}{V_{D, 0} \sqrt{V_{S, 0}+V_{D, 0}}} \\
& \approx \frac{1}{\sqrt{\pi U}} d+O\left(d^{0}\right),
\end{aligned}
$$

which means that we have

$$
\gamma_{D}=\frac{\hbar r_{c}}{96 \pi v_{s, 0} d^{4}},
$$

where $v_{s, 0}=r_{c} \sqrt{\pi n U / m}$ is the sound velocity in an isolated single layer.

\section{SUMMARY AND CONCLUSIONS}

We have studied the dispersions of collective density oscillations in bilayers of ultra-cold Bose gases. Considering different types of inter-particle interactions, we have investigated the long-wavelength behavior of collective modes. For charged bosons, the collective modes are plasmon oscillations and zero sound waves. For dipolar bosons, both modes are of the zero-sound type and the velocity of sound for both in-phase and out-of-phase oscillations is the same within the random phase approximation. Many-body correlations would break the degeneracy of these two modes at strong couplings. ${ }^{24,25}$ For softcore short-range interaction, again both density modes are linear at long wavelengths but the velocity of two modes is different.

Finite counterflow between two layers enhances the energy of symmetric mode and reduces the energy of asymmetric mode. It is expected that at large enough counterflow velocities, the energy of asymmetric mode would become negative, indicating instability of the homogenous gas phase.

Superfluid current in one layer would induce supercurrent in the second layer. The drag coefficient is proportional to $1 / d^{\alpha}$, where $\alpha=7 / 2,5$, and 4, for Coulomb, dipolar and soft-core interaction, respectively.

\section{ACKNOWLEDGMENTS}

S.H.A. acknowledges the hospitality of the Department of Physics at Bilkent University during the early stages of this work. B.T. acknowledges partial support from TUBA.

\section{REFERENCES}

${ }^{1}$ I. Bloch, J. Dalibard, and W. Zwerger, Rev. Mod. Phys. 80, 885 (2008).

${ }^{2}$ S. Giorgini, L. P. Pitaevskii, and S. Stringari, Rev. Mod. Phys. 80, 1215 (2008).

${ }^{3}$ I. Bloch, J. Dalibard, and S. Nascimbène, Nat. Phys. 8, 267 (2012).

${ }^{4}$ M. Randeria and E. Taylor, Annu. Rev. Condens. Matter Phys. 5, 209 (2014).

${ }^{5}$ E. A. Cornell and C. E. Wieman, Rev. Mod. Phys. 74, 875 (2002).

${ }^{6}$ W. Ketterle, Rev. Mod. Phys. 74, 1131 (2002).

${ }^{7}$ G. F. Giuliani and G. Vignale, Quantum Theory of the Electron Liquid (Cambridge University Press, Cambridge, 2005).

${ }^{8}$ A. F. Andreev and E. P. Bashkin, Zh. Éksp. Teor. Fiz. 69, 319 (1975) [Sov. Phys. JETP 42, 164 (1975)].

9.-M. Duan, Phys. Rev. Lett. 70, 3991 (1993).

${ }^{10}$ B. Tanatar and A. K. Das, Phys. Rev. B 54, 13827 (1996).

${ }^{11}$ B. Tanatar, J. Low Temp. Phys. 171, 632 (2013).

${ }^{12}$ S. I. Shevchenko and S. V. Terent'ev, J. Low Temp. Phys. 23, 817 (1997).

${ }^{13}$ D. V. Fil and S. I. Shevchenko, J. Low Temp. Phys. 30, 770 (2004).

${ }^{14}$ D. V. Fil and S. I. Shevchenko, Phys. Rev. A 72, 013616 (2005).

${ }^{15}$ S. I. Shevchenko and D. V. Fil, J. Exp. Theor. Phys. 105, 135 (2007).

${ }^{16}$ L. Y. Kravchenko and D. V. Fil, J. Phys. Condens. Matter 20, 325235 (2008).

${ }^{17}$ M. A. Alpar, S. A. Langer, and J. A. Sauls, Astrophys. J. 282, 533 (1984).

${ }^{18}$ L. Parisi, G. E. Astrakharchik, and S. Giorgini, Phys. Rev. Lett. 121, 025302 (2018).

${ }^{19}$ M. Hobenstreit, M. Rader, and R. E. Zillich, Phys. Rev. A 93, 013611 (2016).

${ }^{20}$ J. K. Block, N. T. Zinner, and G. M. Bruun, New J. Phys. 14, 105006 (2012).

${ }^{21}$ P. Lange, J. Krieg, and P. Kopietz, Phys. Rev. A 93, 033609 (2016).

${ }^{22}$ S. H. Abedinpour, R. Asgari, and M. Polini, Phys. Rev. A 86, 043601 (2012).

${ }^{23}$ I. Seydi, S. H. Abedinpour, R. E. Zillich, R. Asgari, and B. Tanatar, Phys. Rev. A 105, 013628 (2020).

${ }^{24}$ A. Filinov, Phys. Rev. A 94, 013603 (2016).

${ }^{25}$ E. Akaturk, S. H. Abedinpour, and B. Tanatar, J. Phys. Commun. 2, 015018 (2018).

${ }^{\mathbf{2 6}}$ W. Lechner and P. Zoller, Phys. Rev. Lett. 111, 185306 (2013).

Translated by AIP Author Services 